\title{
Effect of the Selected Balance Program on Postural Control of Amputees under Manipulation of Visual, Vestibular and Proprioceptive Systems
}

\section{ART ICLE INF O}

\section{Article Type}

Original Research

\section{Authors}

Mohamadtaghi B.* $M S c$, Hejazi Dinan P. ${ }^{1} P h D$,

Shamsipour Dehkordi P. ${ }^{1} P h D$

How to cite this article
Mohamadtaghi B, Hejazi Dinan P,
Shamsipour Dehkordi P. Effect of
the Selected Balance Program on
Postural Control of Amputees
under Manipulation of Visual,
Vestibular and Proprioceptive
Systems. Iranian Journal of War \&
Public Health. 2016;8(1):1-8.

*Physical Education Department, Physical Education \& Sport Sciences Faculty, Alzahra University, Tehran, Iran

${ }^{1}$ Physical Education Department, Physical Education \& Sport Sciences Faculty, Alzahra University, Tehran, Iran

\section{Correspondence}

Address: No. 2, Abshar Street, Mojen. Shahroud. Postal Code: 3651713543 Phone: +982332573821

Fax: +982188041468

batol.mohamadtaghi@yahoo.com

\section{Article History}

Received: November 17, 2015

Accepted: February 9, 2016

ePublished: April 3, 2016

\section{A B S T RA C T}

Aims Exercise therapy intervening methods are used by physical medicine and rehabilitation therapists to increase the capability to keep balance and independent functioning in the lower limb amputees. The aim of this study was to investigate the effects of the selective balance program on postural control in the lower limb amputees under manipulation of visual, vestibular, and proprioceptive sensory systems.

Materials \& Methods In the semi-experimental study, 14 amputees with under the knee amputation referred to Tehran Red Crescent Rehabilitation Center were studied in .... The subjects selected via available sampling method were divided into two 7-person groups including "control" and "selective postural" groups. Sensory organization test was used to assess postural control and balance of the subjects by computerized dynamic posturography system. Data was analyzed by SPSS 19 software using composed factor ANOVA with repeated measures and Bonferroni post-hoc test.

Findings There was an increase in the balance and postural control in the posttest step in exercise therapy group than control group. In the posttest step in postural group, alignment and strategy scores of the first (presence of visual, vestibular, and proprioceptive senses) and the second (elimination of vision and manipulation of vestibular and proprioceptive senses) situations were higher than other situations, while the score of displacement of the center of gravity in the mentioned situations was less than other situations. In the posttest step in postural group, postural and strategy scores and the score of displacement of the center of gravity in the sixth (manipulation of vestibular and proprioceptive senses) situation were less and higher than other situations, respectively.

Conclusion A course of selective exercise therapy program leads to a reduction in the displacement amplitude and an enhancement in alignment, as well as an increase in strategy, in persons with under the knee amputation, showing increases in balance and postural control.

Keywords Exercise Therapy; Postural Balance; Amputees

\section{CIT A T I O N L IN KS}

Epidemiology of lower extremity amputation in ... [2] Bilateral changes in somatosensory sensibility ... [3] Gender differences in resistance- training- induced myofiber ... [4] Visual effects on people with amputations below the ... [5] Postural reorganization following lower limb amputation ... [6] Equilibrium and movement control strategies in trans-tibial ...

7] Effects of 8 weeks aquatic exercise on static balance in ... [8] Dynamic balance training during standing in people ... [9] Environmental demands associated with ... [10] Postural responses during volitional and perturbed dynamic ... [11] The effect of prosthetic ankle mobility in the sagittal plane on the gait of... [12] Reducing falls in elderly people: A review of exercise ... [13] The maturation of balance in ... [14] Balance in healthy individuals assessed with Equitest: Maturation and normative data for children and ... [15] Age-related changes in postural control sensory ... [16] Beam walking can detect differences in walking balance proficiency across a range of ... [17] Quantitative assessment of ... [18] Effect of balance exercise on balance control in unilateral lower limb ... [19] Antagonism of myostatin enhances muscle regeneration during ... [20] Skeletal muscle myostatin mRNA expression is fiber- type specific and ... [21] Individuals with transtibial limb loss use interlimb force asymmetries to ... [22] Balance control in lower extremity amputees during quiet ... [23] Balance control on a moving platform in ... [24] Visual input: Its importance in the control of postural ... [25] Outcomes after trans-tibial amputation: the relationship between ... [26] Postural changes after sustained neck muscle contraction in persons with ... [27] Attentional demands and postural control: The effect of ... [28] Investigating the validity of ... [29] Aging-induced shifts from the reliance on ... [30] Sensorimotor integration in ... 


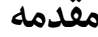

قطع عضو اندام تحتانى كه معمولاً در نتيجه تروما، بيمارىهاى عروقى، ديابت، سرطان، اختلالات مادرزادى و و جراحى ايجاد

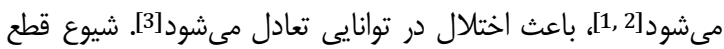

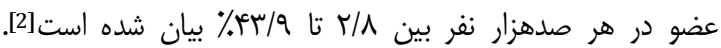

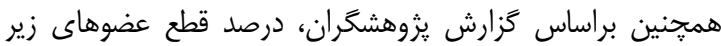

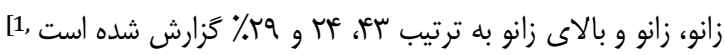

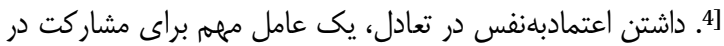

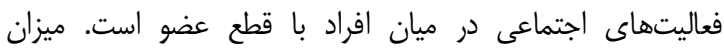

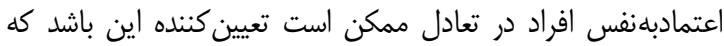

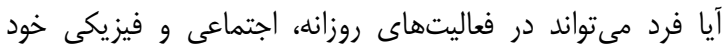

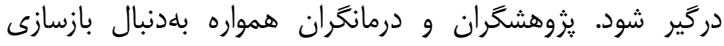
سيستمهاى موثر بر كنترل ياسجر (اطلاعات حسى) افراد قطع عضو دران

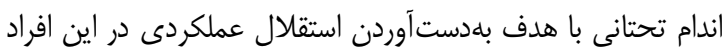

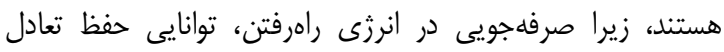

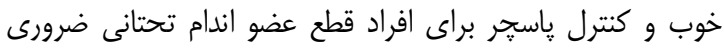
است

مرادى و همكاران، تاثير هشت هفته تمرين در آب را بر تعادل

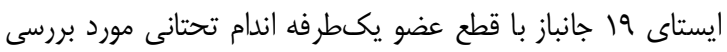

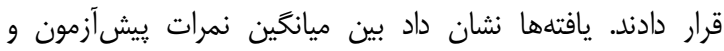

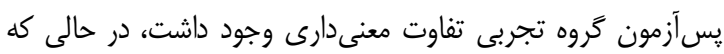

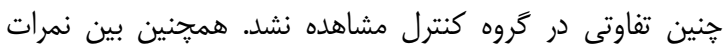

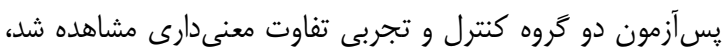

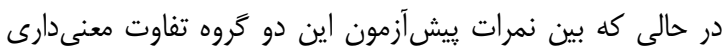

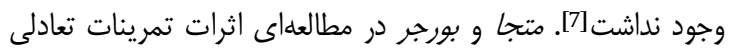
را براى كنترل ياسجر و جلوَّيرى از افتادن در افراد قطع عضو از

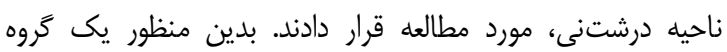

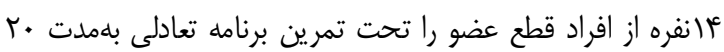
دقيقه، ه روز در هفته قرار دادند. نتايج نشان داد برنامه درمانى

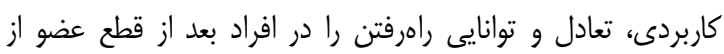

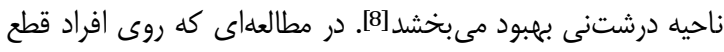

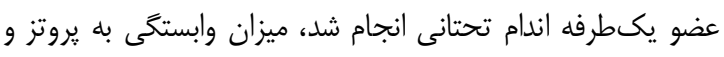

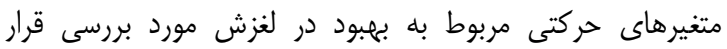

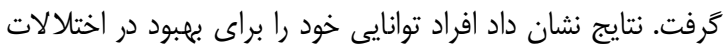

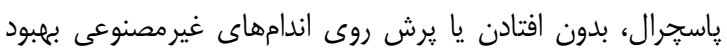

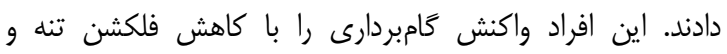

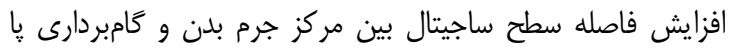

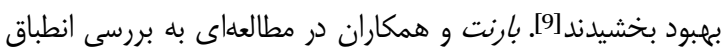

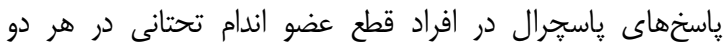

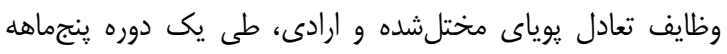

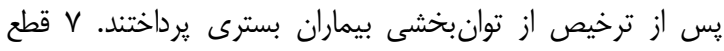

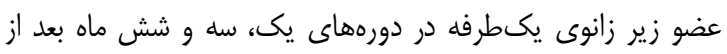
دوره ^، شماره ا، زمستان ITFF
تاثير برنامه منتخب تعادلى بر كنترل ياسجٍر

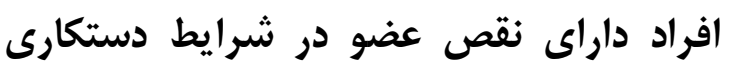
سيستمهاى بينايى، دهليزى و عمقى MSc بتول محمدتقى كروه رفتار حركتى، دانشكده تربيت بدنى و علوم ورزشى، دانشعاه الزهرا، تهران، ايران

تريسا حجازى دينان PhD گروه رفتار حركتى، دانشكده تربيت بدان بدنى و علوم ورزشى، دانشخاه الزهرا، تهران، ايران

يروانه شمسى يور دهكردى PhD كرّوه رفتار حركتى، دانشكده تربيت بدنى و و علوم ورزشى، دانشعاه الزهرا، تهران، ايران

جكيده

اهداف: درمانگران طب فيزيكى و توانبخشى براى افزايش توانايى حفظ تعادل و ارتقاى استقلال عملكردى در افراد با قطع عضو اندام تحتانى، از روشهاى مداخلهاى تمريندرمانى استفاده مى كنند. هدف تحقيق حاضر،

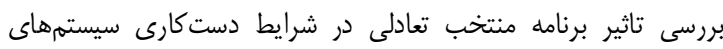

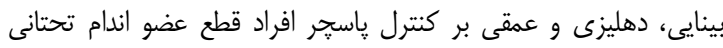

بود. - مان مواد و روشها: در اين يزوهش نيمهتجربى در سال عوسا، عال نفر با زيا

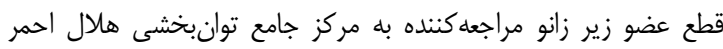

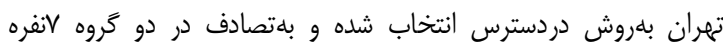

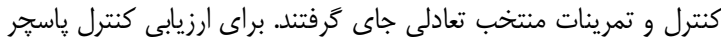

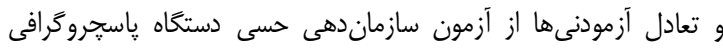

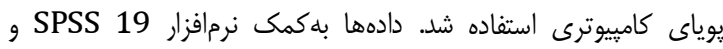
توسط آزمون تحليل واريانس عاملى تركيبى با اندازههاى تكرارى و آزمون تعقيبى بونفرونى تحليل شدند.

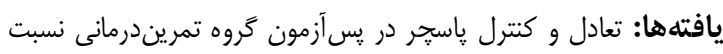

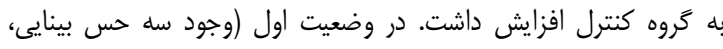

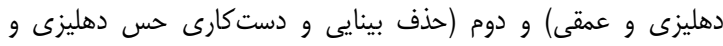

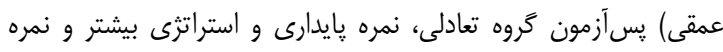

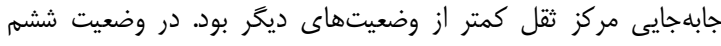

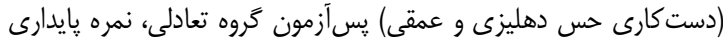

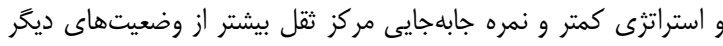

بود.

نتيجه كَيرى: انجام يك دوره برنامه منتخب تمريندرمانى باعث كاهش

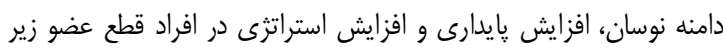

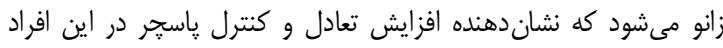

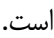
كليدوازهها: تمريندرمانى، اطلاعات حسى، كنترل پاسحر، قطع عضو زير زانو

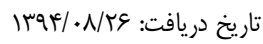

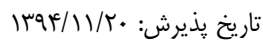
"نويسنده مسئول: batol.mohamadtaghi@yahoo.com 
تاثير برنامه منتخب تعادلى بر كنترل ياسجر افراد داراى نقص عضو در شرايط دستكارى سيستمهاى بينايى، دهليزى و عمقى

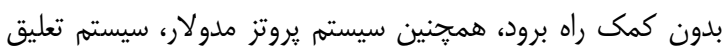

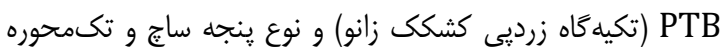

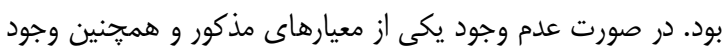

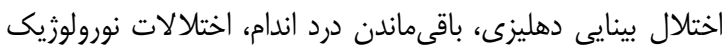

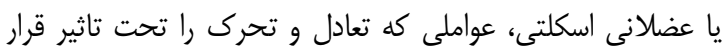

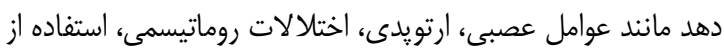

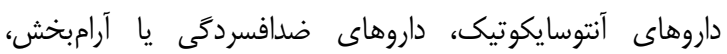
شركت كنندهها از مطالعه خارج مىشدائد.

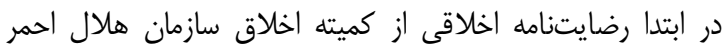

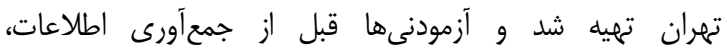
رضايتنامه شركت در آزمون را امضا كردند. سيس در فر يك جلى جلسه نحوه انجام آزمونها، تمرينات و در نهايت خلاصهایى از تحقيق

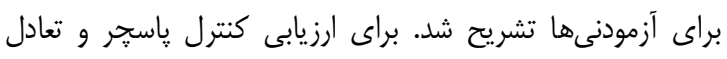

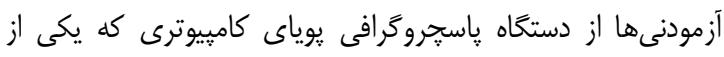
ييشرفتهترين سيستمهاى بررسى و دست كارى سيستمهاى حسى ديى

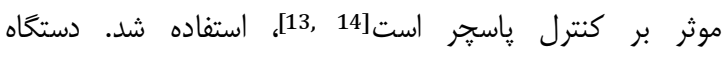

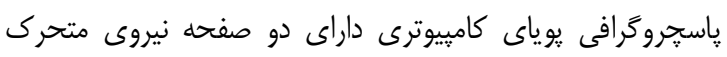

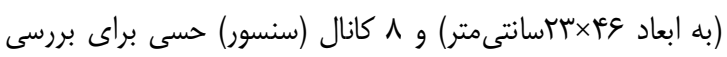

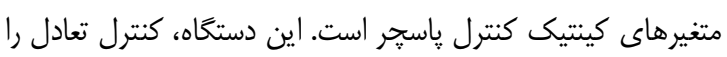

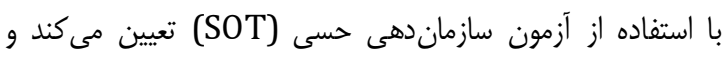

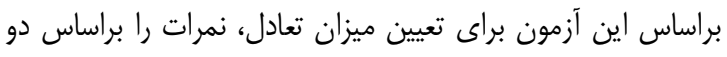

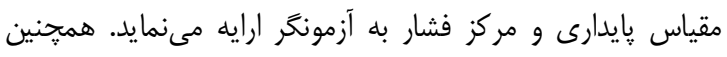

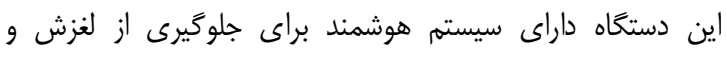

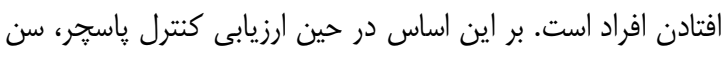

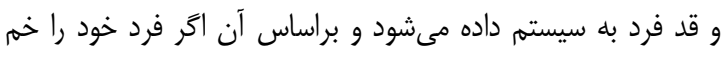

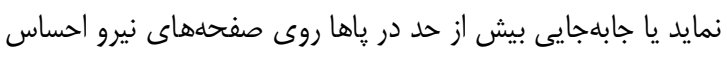
شود، سيستم بلطور خودكار متوقف مى شود.

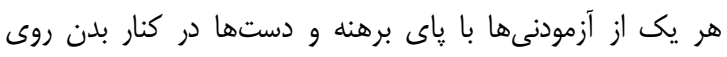

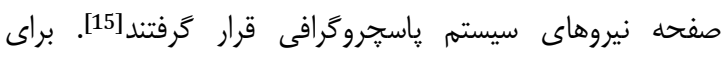

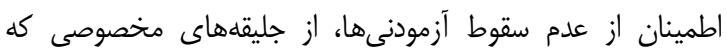

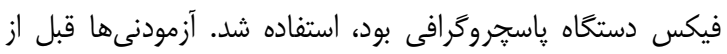

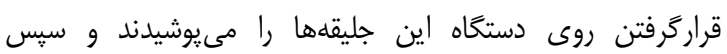

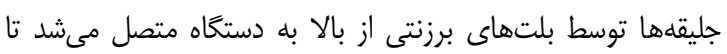

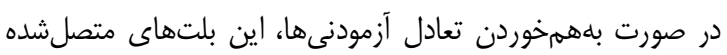

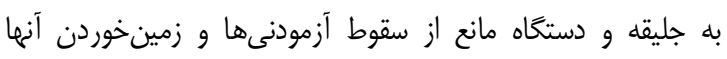

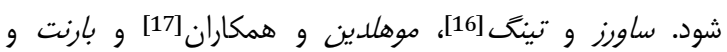

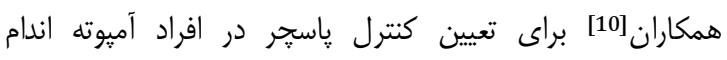

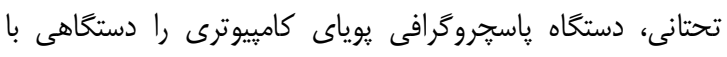

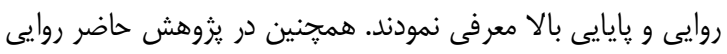

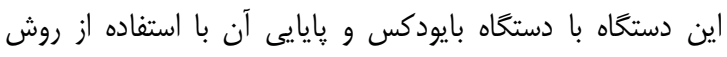

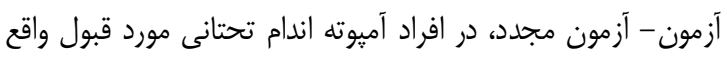

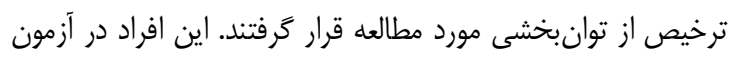

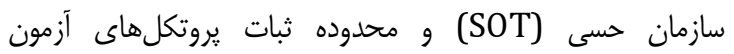

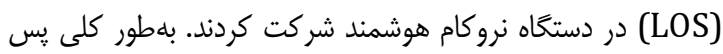
از ترخيص، توانايى تعادل بهبود قابل توجهى را در استفاده از ورودى نرى حسى عمقى و كاهش استراتثى ران نشان داد. زمان واكنش و و سرعت حركت در اكثر جهتهاى هدف براى آزمون LOS تغيير

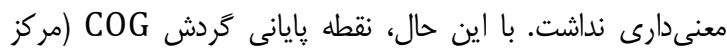

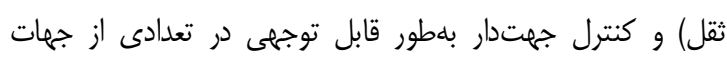

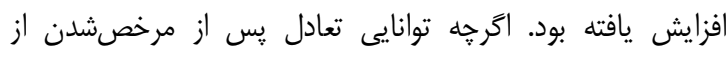

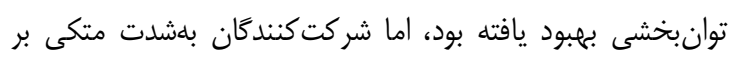

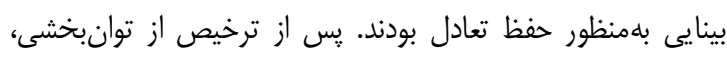

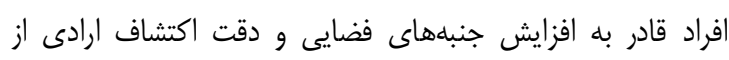

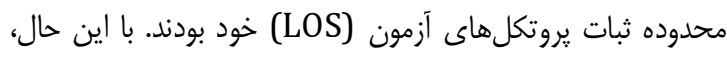

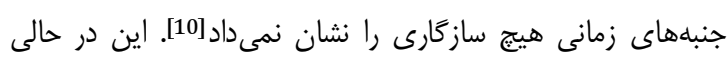

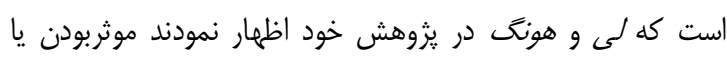

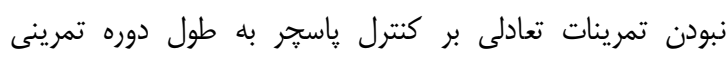
بستخى دارد [11].

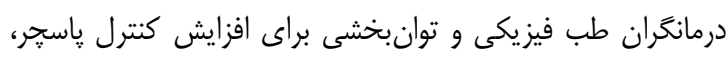

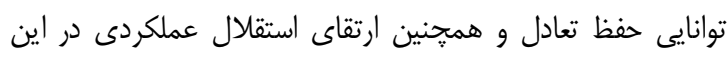

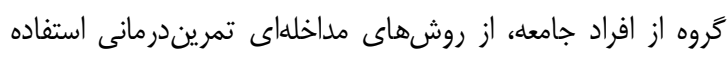

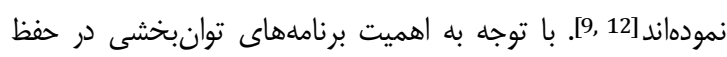

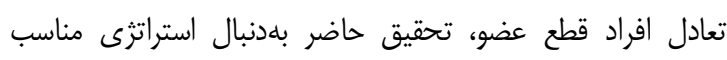

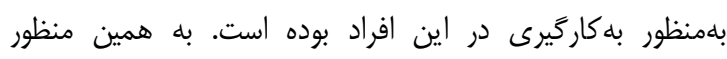

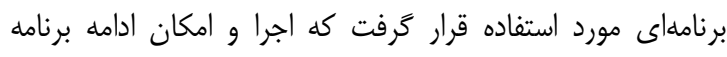

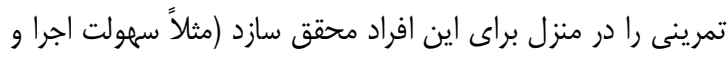

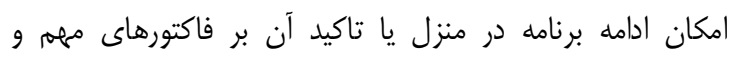
اثربخش). هدف تحقيق حاضر، بررسى تاثير برنامه منتخب تعادلى در شرايط

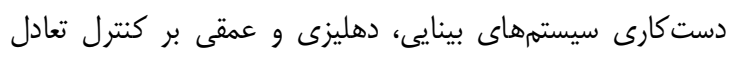
افراد قطع عضو اندام تحتانى بود.

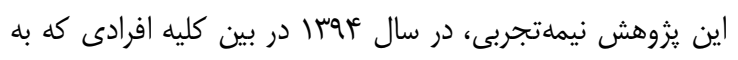

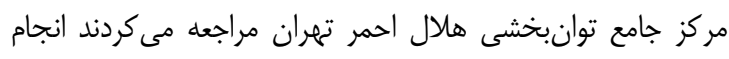

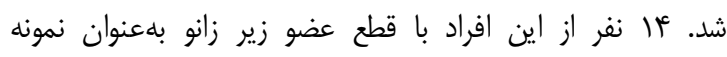

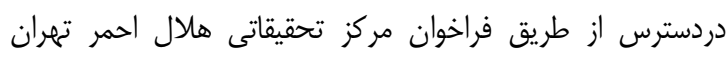

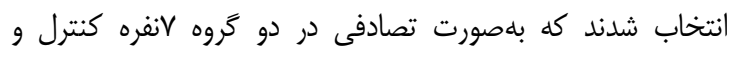

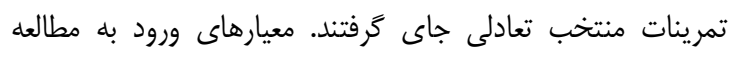

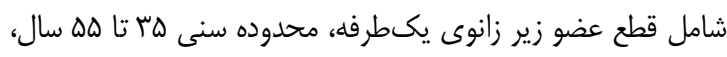

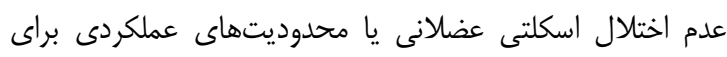

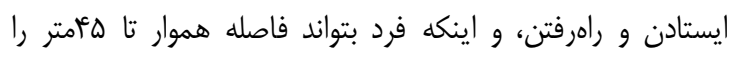


جابهجايى مركز ثقل بهعنوان متغير وابسته مورد بررسى قرار كرفتنند.

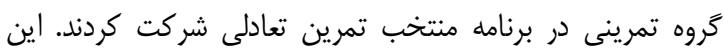

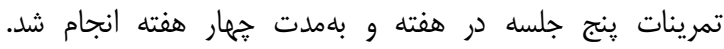
تمرينات بروتكل شامل • ا تمرين متفاوت تعادلى بود كه مطابق بات بات

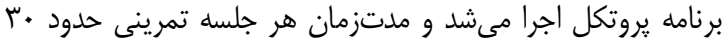

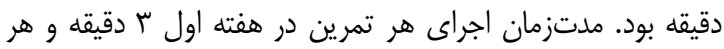

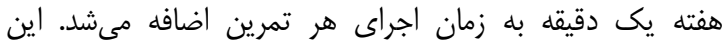

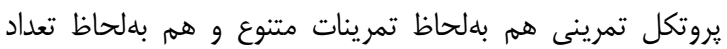

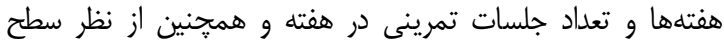

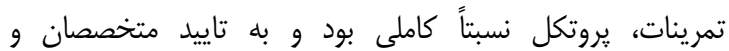

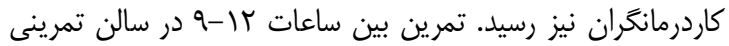

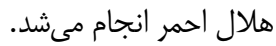

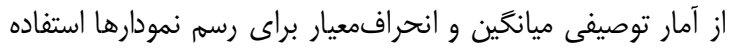

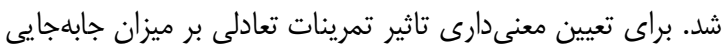

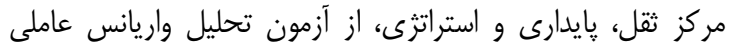

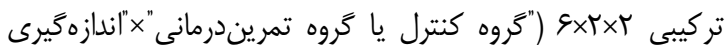

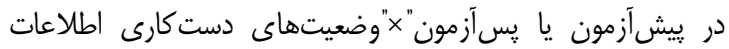

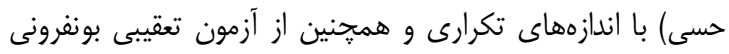

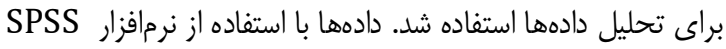

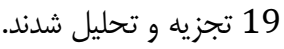

در تحقيق از آزمون سازماندهى حسى استى استفاده شد. اين آزمون

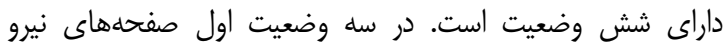
ثابت هستند و در سه وضعيت ديكر در جهتهاي قداى قدامى و خلفى

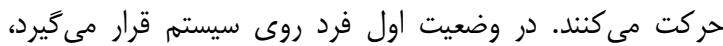

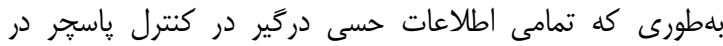

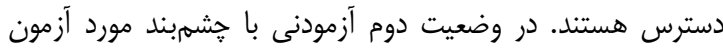

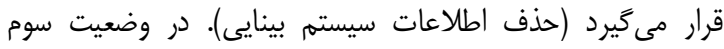

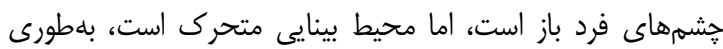

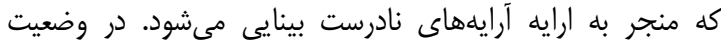
تجهارم صفحههاى نيرو متحرى هستند و اطلاعات حس عمقى نقى

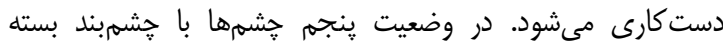
مىشوند و صفحه نيروى متحرى نيز باعث دست اكارى اطلاعات حس عمقى مى شود. در اين وضعيت اطلاعات سيستم دهليزى در

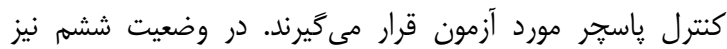
اطلاعات دردسترس حس بينايى حذف شده و اطلاعات حسهاى

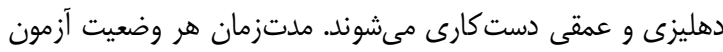

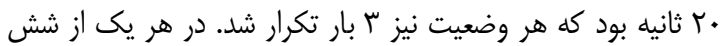
وضعيت اين آزمون، نمره صفر تا ..1 بلعنوان شاخص كنترل تعادل فرد ارايه مىشود[14]. تعديل اطلاعات آوران حسى (بينايى،

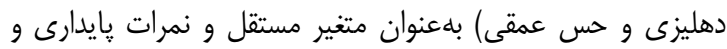

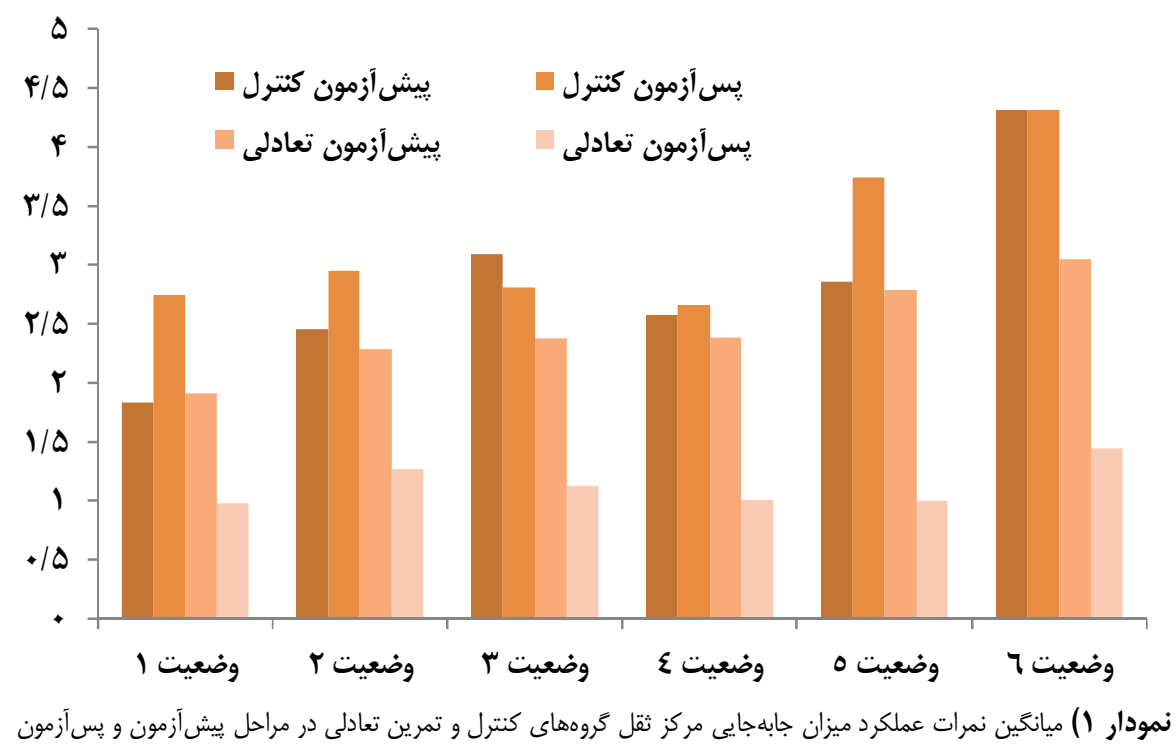

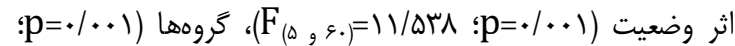

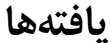

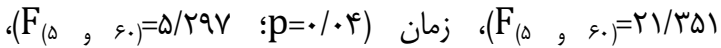
"وضعيت كَروها" (

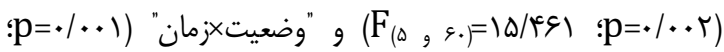

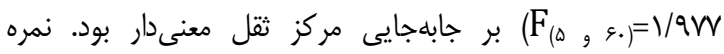
جابهجايى مركز ثقل در كروه تعادلى (با ميانكَين روايى دستخاه بايودكس با استفاده از ضريب همبستخى ييرسون

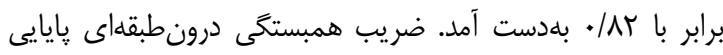
دستخاه نيز در دوبار اندازهيرى تعادل در افراد آميوته اندام تحتانى درئي

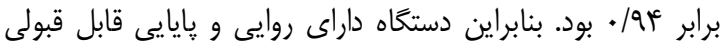
براى مورد استفاده قراركرفتن در افراد قطع عضو اندام تحتانى بود. 


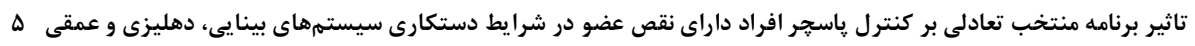

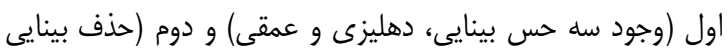
و وجود دو حس دهليزى و عمقى) عملكرد بهترى نسبت بله ساير

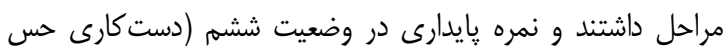
دهليزى و عمقى) كمتر از وضعيتهاى ديخر بود (نمودار بار). اثر وضعيت (1)

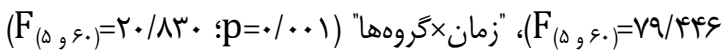

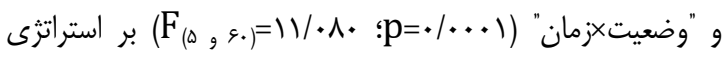

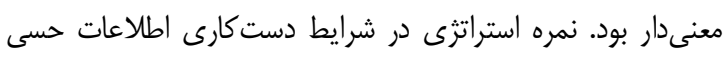

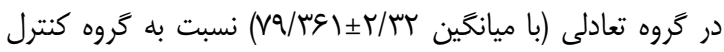

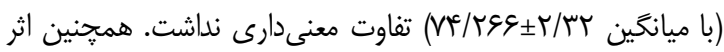

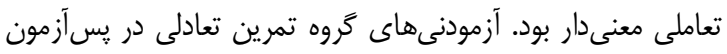

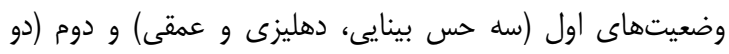
حس دهليزى و عمقى) عملكرد بهترى نسبت به ساير مراحل

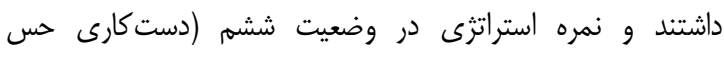
دهليزى و عمقى) كمتر از وضعيتهاى ديخر بود (نمودار سا).

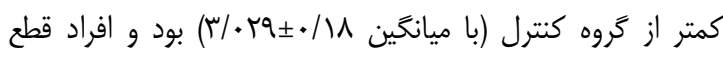
عضو اندام تحتانى در رَوه تعادلى نوسان كمتر و تعادل بهتر نسبت

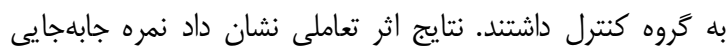

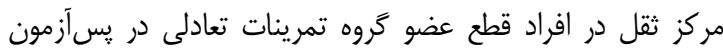
وضعيت اول (وجود حس بينايى، عمقى و دهليزى) و وضعيت دوم

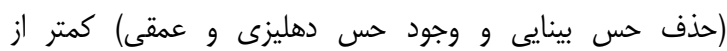

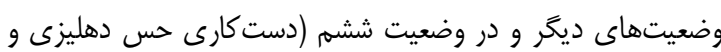
عمقى) بيشتر از وضعيتهاى ديخر بود (نمودار ().

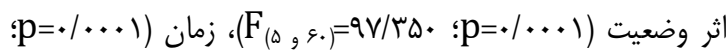

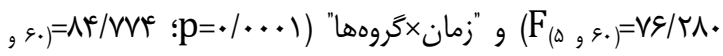

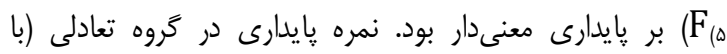

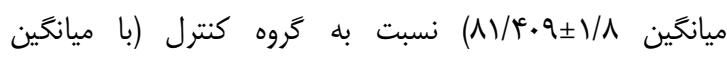

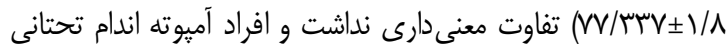

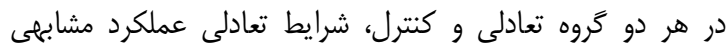

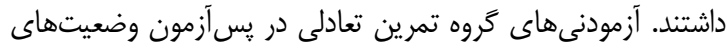

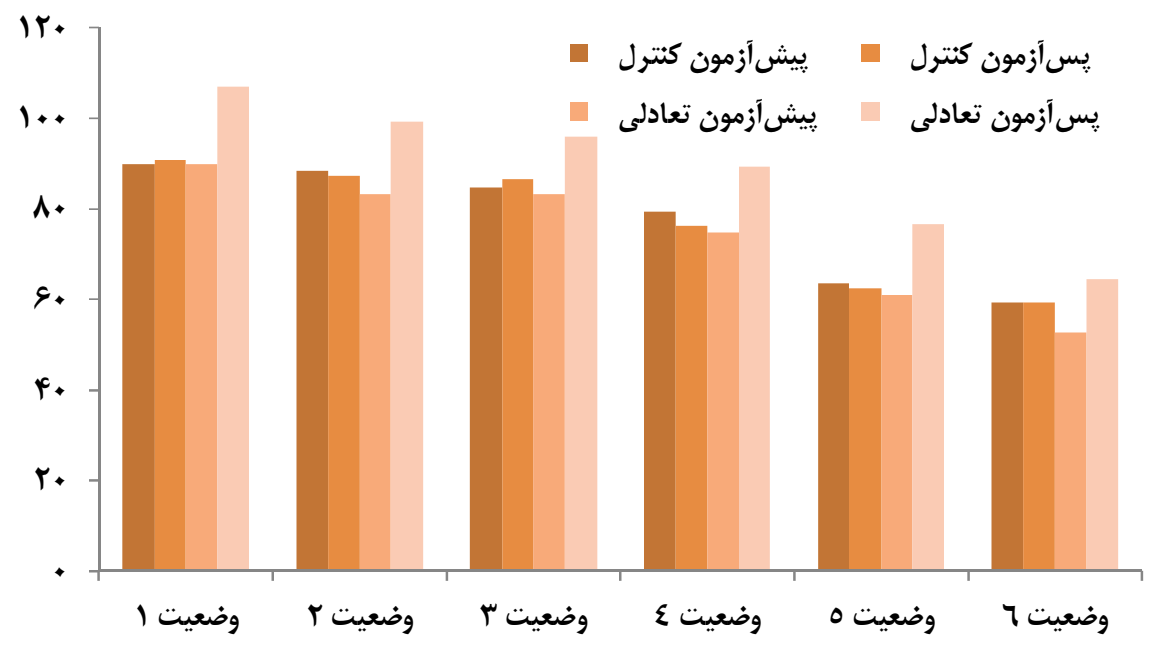

نمودار r) ميانكين نمرات عملكرد ميزان پايدارى كَروهاى كنترل و تمرين تعادلى در مراحل بيش آزمون و پِّآزمون

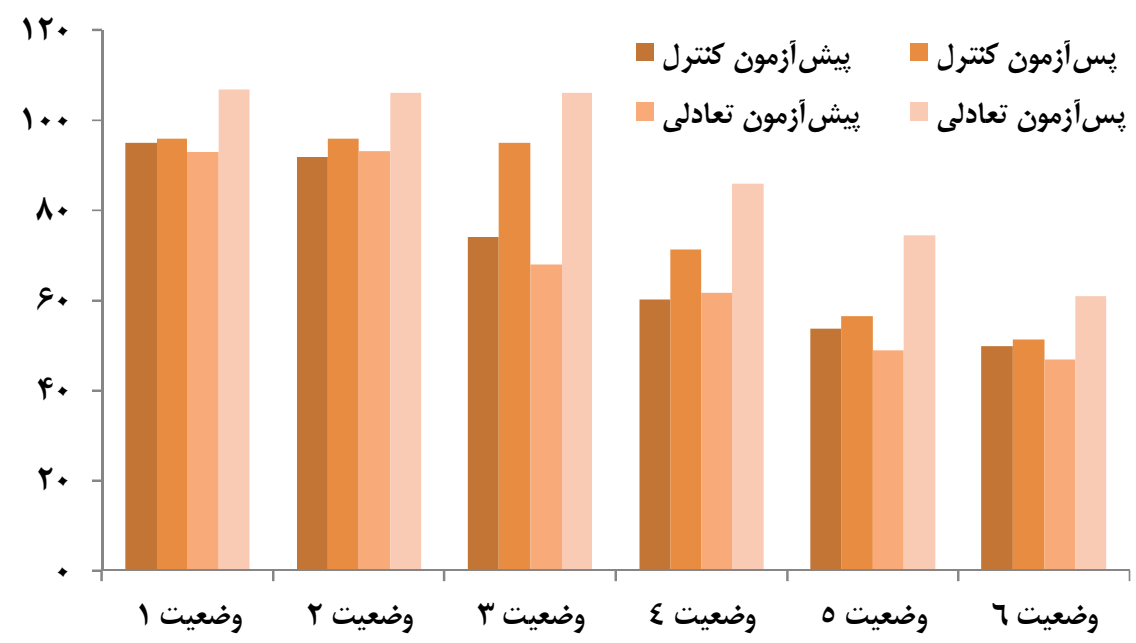

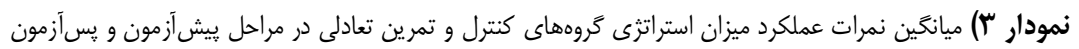


يافتهاى درنان و همكاران [24] كه بيان داشتند با افزايش درصد

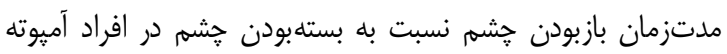

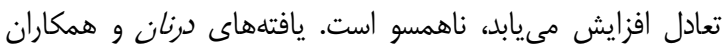

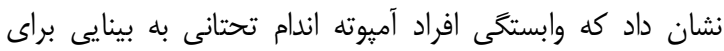

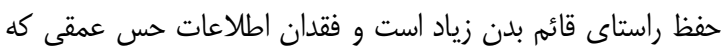

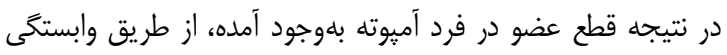

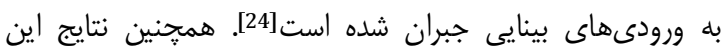

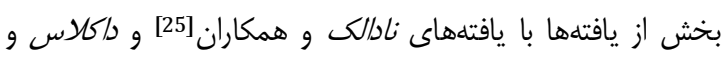

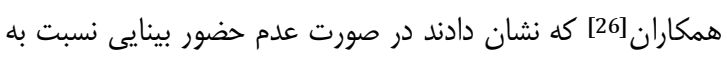

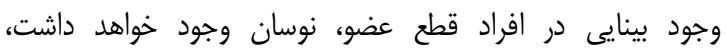

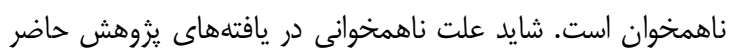

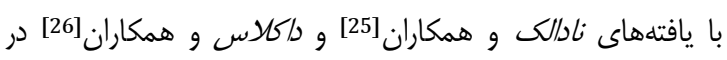

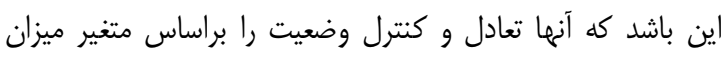

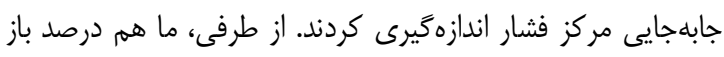

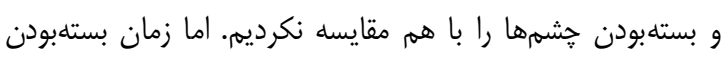

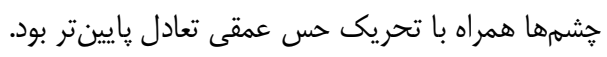

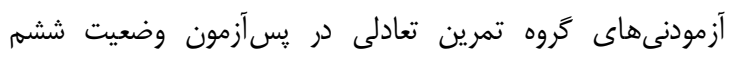

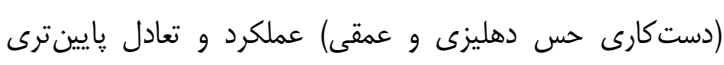

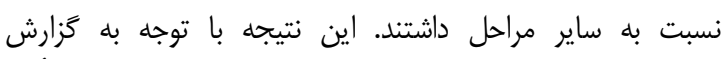

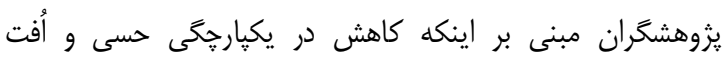

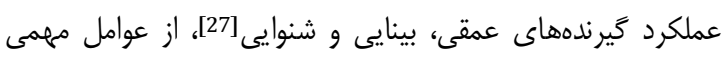

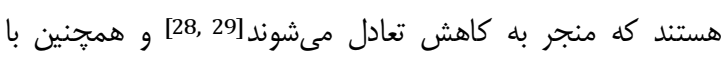

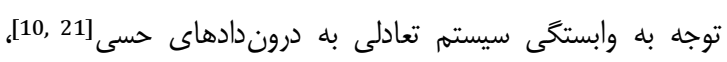

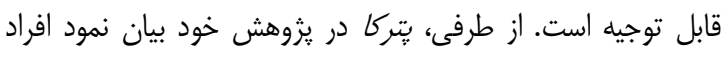

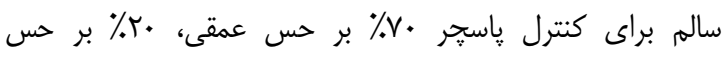

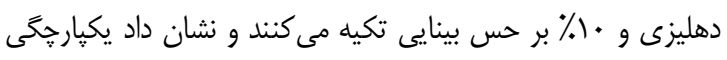

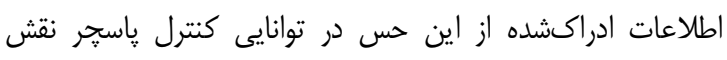
اساسى بازى مى كند[30].

در مورد محدوديتهاى اين مطالعه، بايد يادآور شويم كه تنها

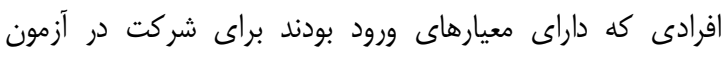

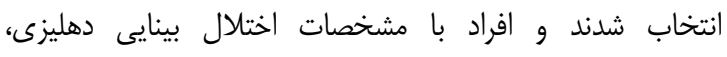

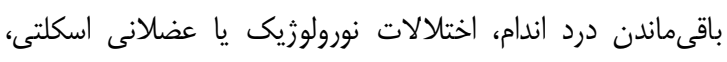

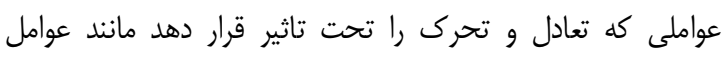

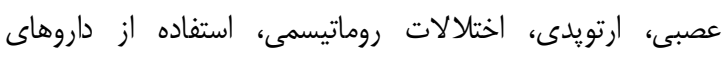

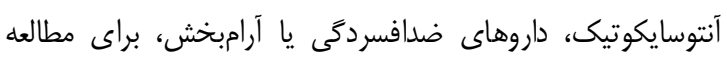
مورد تاييد واقع نشدند.

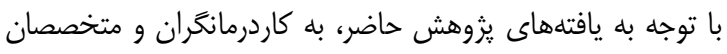

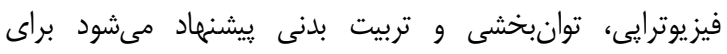

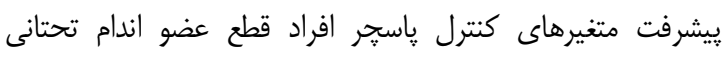

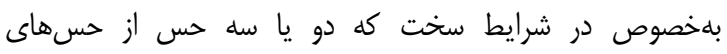

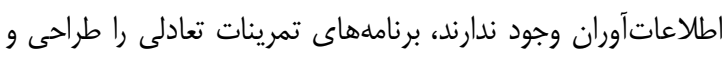

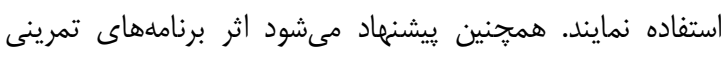
دوره ^، شماره ا، زمستان Iraf

\section{بحث}

هدف از مطالعه حاضر، بررسى تاثير برنامه منتخب تعادلى در شرايط

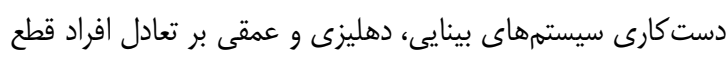

عضو بود.

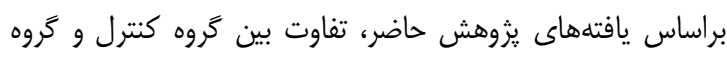

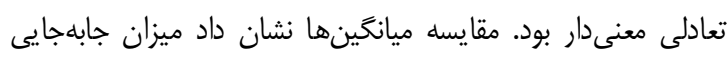

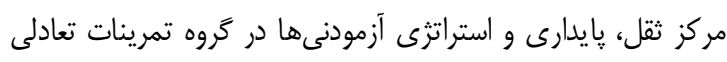

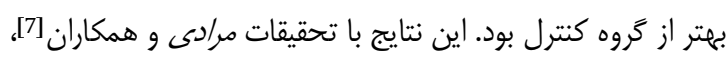

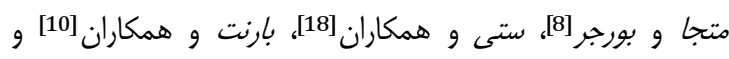
كمالى و همكاران [4] همسو بود.

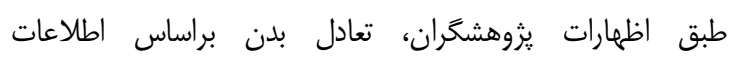

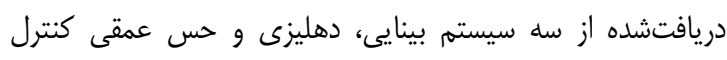

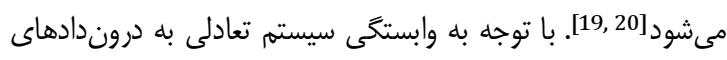

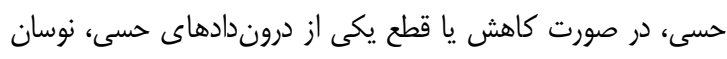

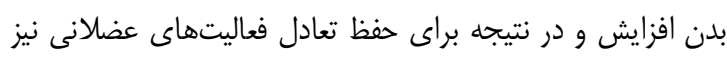

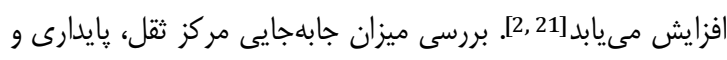

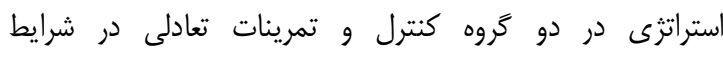

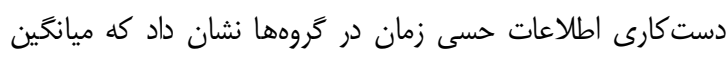

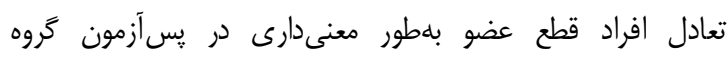

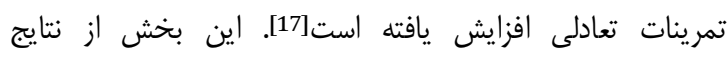

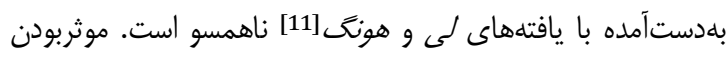

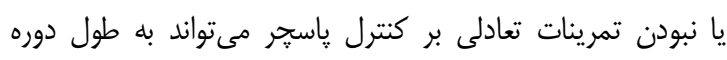

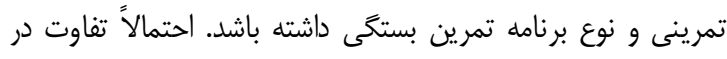

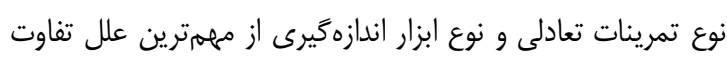

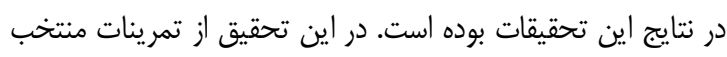

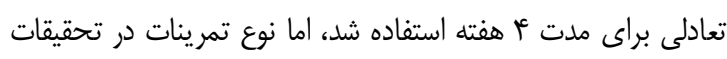

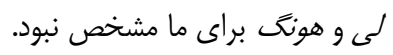

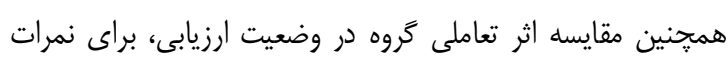

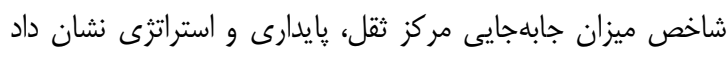

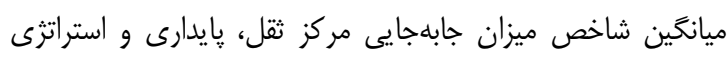

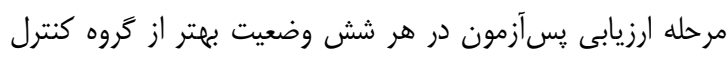

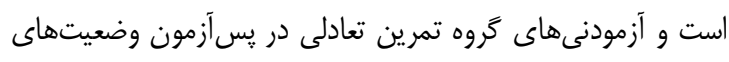

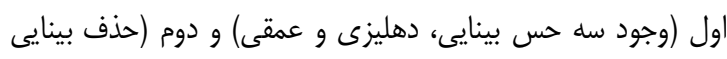

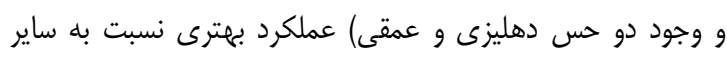

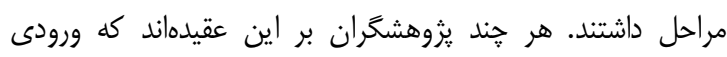

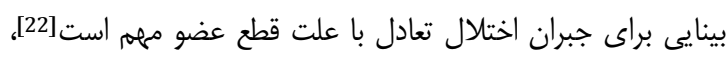

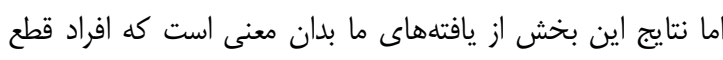

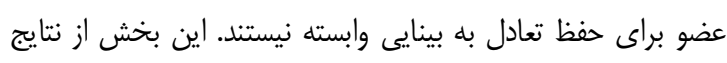

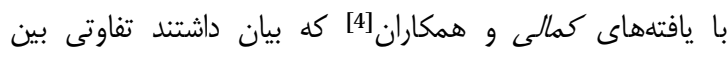

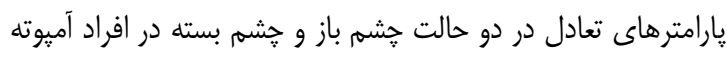

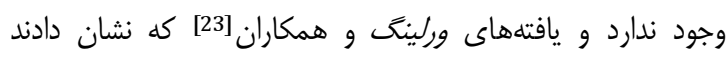

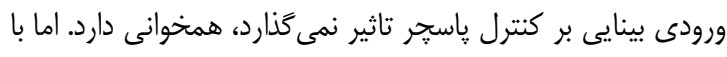
فصل نامه علمى - يزووهشى طب جانباز 
تاثير برنامه منتخب تعادلى بر كنترل ياسجر افراد داراى نقص عضو در شرايط دستكارى سيستمهاى بينايى، دهليزى و عمقى

9- Shumway-Cook A, Patla AE, Stewart A, Ferrucci L, Ciol MA, Guralnik JM. Environmental demands associated with community mobility in older adults with and without mobility disabilities. Phys Ther. 2002;82(7):67081.

10- Barnett CT, Vanicek N, Polman RC. Postural responses during volitional and perturbed dynamic balance tasks in new lower limb amputees: A longitudinal study. Gait Posture. 2013;37(3):319-25.

11- Lee S, Hong J. The effect of prosthetic ankle mobility in the sagittal plane on the gait of transfemoral amputees wearing a stance phase controlled knee prosthesis. Proc Inst Mech Eng H. 2009;223(2):263-71.

12- Brown AP. Reducing falls in elderly people: A review of exercise interventions. J Phys Ther. 1999;15(2):59-68.

13- Cumberworth VL, Patel NN, Rogers W, Kenyon GS. The maturation of balance in children. J Laryngol Otol. 2007;121(5):449-54.

14- Ferber-Viart C, Ionescu E, Morlet T, Froehlich P, Dubreuil C. Balance in healthy individuals assessed with Equitest: Maturation and normative data for children and young adults. Int J Pediatr Otorhinolaryngol. 2007;71(7):1041-6.

15- Rinaldi NM, Polastri PF, Barela JA. Age-related changes in postural control sensory reweighting. Neurosci Lett. 2009;467(3):225-9.

16- Sawers A, Ting LH. Beam walking can detect differences in walking balance proficiency across a range of sensorimotor abilities. Gait Posture. 2015;41(2):61923.

17- Mohieldin AM, Ambalavanan C, Ramar S, Waleed AB. Quantitative assessment of postural stability and balance between persons with lower limb amputation and normal subjects by using dynamic posturography. Mac J Med Sci. 2010;3(2):138-43.

18- Sethy D, Kujur ES, Sau K. Effect of balance exercise on balance control in unilateral lower limb amputees. Indian J Occup Ther. 2009;7(3):63-8.

19- Siriett V, Salerno MS, Berry C, Nicholas G, Bower R, Kambadur R, et al. Antagonism of myostatin enhances muscle regeneration during sarcopenia. Mol Ther. 2007;15(8):1463-70.

20- Carlson CJ, Booth FW, Gordon SE. Skeletal muscle myostatin mRNA expression is fiber- type specific and increases during hindlimb unloading. Am J Physiol. 1999;277(2 Pt 2):R601-6.

21- Bolger D, Ting LH, Sawers A. Individuals with transtibial limb loss use interlimb force asymmetries to maintain multi-directional reactive balance control. Clinical Biomechanics. 2014;29(9):1039-47.

22- Ku PX, Abu Osman NA, Wan Abas WA. Balance control in lower extremity amputees during quiet standing: A systematic review. Gait Posture. 2014;39(2):672-82.

23- Vrieling AH, van Keeken HG, Schoppen T, Otten E, Hof AL, Halbertsma JP, et al. Balance control on a moving platform in unilateral lower limb amputees. Gait Posture. 2008;28(2):222-8.

24- Dornan J, Fernie GR, Holliday PJ. Visual input: Its importance in the control of postural sway. Arch Phys Med Rehabil. 1978;59(12):586-91.

25- Nadollek H, Brauer S. Isles R. Outcomes after transtibial amputation: the relationship between quiet stance ability, strength of hip abductor muscles and gait. Physiother Res Int. 2002;7(4):203-14.

26- Duclos C, Roll R, Kavounoudias A, Mongeau JP, Roll JP, Forget R. Postural changes after sustained neck

$$
\begin{aligned}
& \text { منتخب ديخر مثل تمرينات قدرتى، استقامتى و انعطافيذيرى يا }
\end{aligned}
$$

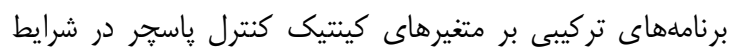

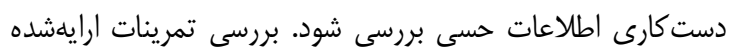

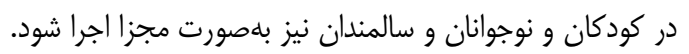

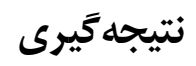

انجام يك دوره برنامه منتخب تمريندرمانى باعث كاهش دامنه

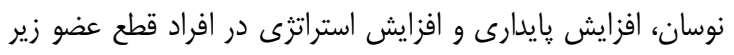

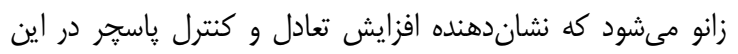

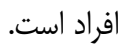

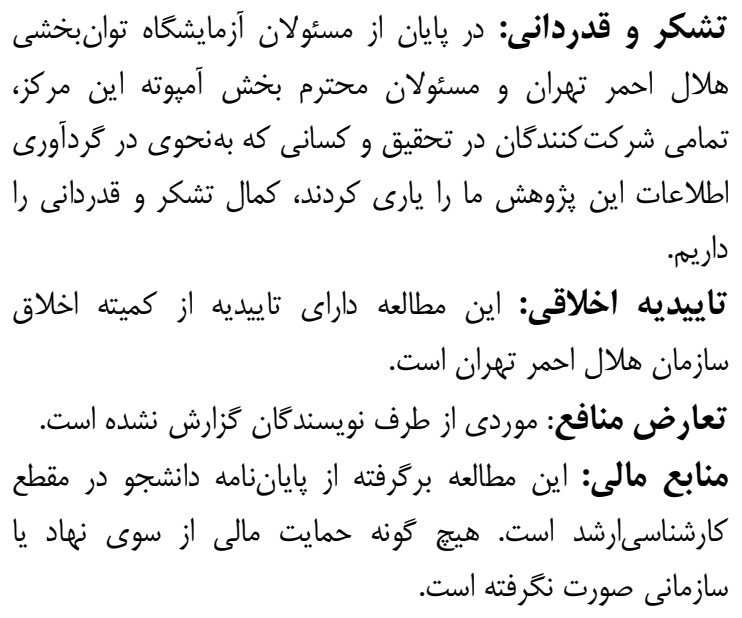

منابع

1- Unwin N. Epidemiology of lower extremity amputation in centres in Europe, North America and East Asia. Br J Surg. 2000;87(3):328-37.

2- Kavounoudias A, Tremblay C, Gravel D, Iancu A, Forget R. Bilateral changes in somatosensory sensibility after unilateral below-knee amputation. Arch Phys Med Rehabil. 2005;86(4):633-40.

3- Bamman MM, Hill VJ, Adams GR, Haddad F, Wetzstein CJ, Gower BA, et al. Gender differences in resistancetraining- induced myofiber hypertrophy among older adults. J Gerontol A Biol Sci Med Sci. 2003;58(2):108-16.

4- Kamali M, Qaderi M, Karimi M. Visual effects on people with amputations below the knee Station balance. Urmia Med J. 2014;25(9):845-52.

5- Geurts AC, Mulder TW, Nienhuis B, Rijken RA. Postural reorganization following lower limb amputation Possible motor and sensory determinants of recovery. Scand J Rehabil Med. 1992;24(2):83-90.

6- Viton JM1, Mouchnino L, Mille ML, Cincera M, Delarque A, Pedotti A, et al. Equilibrium and movement control strategies in trans-tibial amputees. Prosthet Orthot Int. 2000;24(2):108-16.

7- Moradi Y, Behpoor N, Ghaeeni S, Shamsakohan P. Effects of 8 weeks aquatic exercise on static balance in veterans with unilateral lower limb amputation. Iran J War Public Health. 2014;6(2):27-34. [Persian]

8- Matjaĉić Z, Burger H. Dynamic balance training during standing in people with trans-tibial amputation: A pilot study. Prosthet Orthot Int. 2003;27(3):214-20. 
framework underlying the original and modified Dynamic Gait Index. Phys Ther. 2015;95(6):864-70.

29- Benjuya A, Melzer I, Kaplanski J. Aging-induced shifts from the reliance on sensory input to muscle cocontraction during balanced standing. J Gerontol A Biol Sci Med Sci. 2004;59(2):166-71.

30- Peterka RJ. Sensorimotor integration in human postural control. J Neurophysiol. 2002;88(3):1097-118. muscle contraction in persons with a lower leg amputation. J Electromyogr Kinesiol. 2009;19(4):e21422.

27- Shumway-Cook A, Woollacott M. Attentional demands and postural control: The effect of sensory context. J Gerontol A Biol Sci Med Sci. 2000;55(1):M10-6. 28- Shumway-Cook A, Matsuda PN, Taylor C. Investigating the validity of the environmental 